\title{
Erste Pilotstudien aus China zum Einsatz von Rekonvaleszentenplasma bei COVID-19
}

Duan K et al. Effectiveness of convalescent plasma therapy in severe COVID-19 patients. Proc Natl Acad Sci U S A 2020; 117: 9490-9496

Shen C et al. Treatment of 5 Critically III Patients with COVID-19 with Convalescent Plasma. JAMA 2020; 323: 1582-1589

Duan et al. untersuchten Aspekte der Plasmatransfusion bei 10 schwer an COVID-19 Erkrankten, Shen et al. gingen anhand einer Fallserie von 5 kritisch kranken COVID-19-Patienten und akutem Atemnotsyndrom (ARDS) der Frage nach, inwieweit Plasma Rekonvaleszenter die Therapie günstig beeinflusst. Eine einmalige Plasmatransfusion wurde jeweils gut toleriert, eine signifikante Verbesserung der klinischen Symptome ließ sich in beiden Studien nachweisen.

\section{Fallserie von 10 Patienten aus Wuhan}

Die prospektive Pilotstudie von Duan et al. wurde von Januar bis Februar 2020 an 3 Kliniken in Wuhan, China, durchgeführt, eingeschlossen wurden 10 Patienten, Einschlusskriterien waren Alter $\geq 18$ Jahre und

- Atemnot, Atemfrequenz $\geq 30$ /Minute und/oder

- Sauerstoffsättigung $<93 \%$ in Ruhe und/oder 
- Sauerstoffpartialdruck $\left(\mathrm{PaO}_{2}\right) /$ Sauerstoffkonzentration $\left(\mathrm{FiO}_{2}\right) \leq 300 \mathrm{mmHg}$ $(1 \mathrm{mmHg}=0,133 \mathrm{kPa})$.

Die Patienten (6 männlich, 4 weiblich) waren im Median 52,5 Jahre alt. Einschlusskriterien für die Plasmaspender waren Fieberfreiheit für > 3 Tage, keine Atemwegssymptome mehr, einen Titer neutralisierender Antikörper von > 1:640 und 2 aufeinanderfolgende negative Ergebnisse im Sputum hinsichtlich SARS-CoV-2 durch einen RT-PCR-Assay (1 Tag Intervall). Die Abnahme der Plasmaspende (Convalescent Plasma [CP]) erfolgte 3 Wochen nach Krankheitsbeginn und 4 Tage nach Krankenhausentlassung.

Primärer Endpunkt war die Sicherheit einer CP-Transfusion. Sekundäre Endpunkte waren die Verbesserung klinischer Symptome und radiologischer und Laborparameter innerhalb von 3 Tagen nach CP-Transfusion.

Die mediane Zeit von Symptombeginn bis Krankenhausaufnahme war 6 Tage (IQR 2,5-8,5) und bis zur CP-Transfusion 16,5 Tage (IQR 11,0-19,3). Vier Patienten wiesen Vorerkrankungen auf, einschließlich kardiovaskulärer und/oder zerebrovaskulärer Erkrankungen sowie essenzieller arterieller Hypertonie. Neun Patienten erhielten Arbidol ${ }^{\circledR}$ (Umifenovir) entweder als Monotherapie oder in Kombination mit Remdesivir, Ribavirin oder Peramivir, 1 Patient erhielt eine Ribavirin-Monotherapie, 6 Patienten erhielten Methylprednisolon $20 \mathrm{mg}$ i. v. alle $24 \mathrm{~h}$.

Alle eingeschlossenen Patienten wiesen im CT unter anderem eine bilaterale Milchglastrübung auf und/oder pulmonalparenchymale Verdichtungen mit vorwiegend subpleuralem und bronchiovaskulärem Verteilungsmuster. Drei Patienten mussten mechanisch beatmet werden.

Nach der CP-Transfusion (jeweils $200 \mathrm{ml}$ ) stieg der Spiegel der neutralisierenden Antikörper rasch an auf 1:640 bei 5 Patienten, der Spiegel bei den anderen 4 Patienten blieb stabil bei $1: 640$, bei 1 Patient war der Titer nicht bestimmt worden. Die klinischen Symptome verbesserten sich signifikant und gingen einher mit einem
Anstieg der Oxyhämoglobinsättigung innerhalb von 3 Tagen.

Die $\mathrm{SaO}_{2}$ nahm von im Median 93 auf 96\% zu, radiologische Bildgebungen zeigten ein variierendes Ausmaß an Absorption pulmonaler Läsionen innerhalb von 7 Tagen. Eine Viruslast war nach Transfusion bei 7 Patienten nicht mehr messbar. Schwere unerwünschte Ereignisse wurden nicht beobachtet.

Eine Kontrollgruppe von 10 Patienten derselben Kliniken mit vergleichbaren Aufnahmecharakteristika wurde gebildet. In der CP-Gruppe konnten 3 Patienten nach Hause entlassen werden, während $7 \mathrm{~Pa}$ tienten in deutlich verbessertem Allgemeinzustand vor Entlassung standen. In der Kontrollgruppe waren dahingegen 3 Patienten verstorben, 6 Patienten konnten stabilisiert werden und 1 Patient war in verbessertem Allgemeinzustand.

\section{Fallserie von 5 Patienten aus Shenzhen}

Shen et al. untersuchten 5 Patienten von Januar bis März 2020 in Shenzhen, China. Einschlusskriterien beinhalteten das Auftreten einer schweren Pneumonie mit rascher Verschlechterung und anhaltend hoher Viruslast trotz antiviraler Therapie (einschließlich Interferon und Lopinavir/Ritonavir), ferner ein $\mathrm{PaO}_{2} / \mathrm{FiO}_{2}<300 \mathrm{mmHg}$ und mechanische Beatmung. Alle 5 Patienten erhielten zudem zum Zeitpunkt der Transfusion Methylprednisolon.

Die Patienten (36-73 Jahre alt, 2 weiblich) waren jeweils Nichtraucher, 4 von 5 wiesen keine Vorerkrankungen auf. Die Plasmatransfusion wurde jeweils 10-22 Tage nach Krankenhausaufnahme durchgeführt. Jeder Patient erhielt 2 Transfusionen AB0-kompatibles Rekonvaleszentenplasma am selben Tag wie von dem jeweiligen Spender entnommen (insgesamt jeweils $400 \mathrm{ml}$ ). Die antivirale Medikation wurde parallel beibehalten, bis die SARS-CoV-2Viruslast negativ wurde.

Die 5 Spender waren 18-60 Jahre alt, seit mindestens 10 Tagen vor der Spende beschwerdefrei und wiesen einen SARS-CoV-2-spezifischen ELISA-AK-Titer
$>$ 1:1000 und einen Neutralisationstiter $>40$ auf.

Nach der Transfusion

- normalisierte sich die Körpertemperatur innerhalb von 3 Tagen bei 4 von 5 Patienten,

- nahm der SOFA-Score (Sequential Organ Failure Assessment) ab (2-10 vor der Transfusion, 12 Tage post transfusionem 1-4)

- und stieg das Verhältnis $\mathrm{PaO}_{2} / \mathrm{FiO}_{2}$ innerhalb von 12 Tagen von 172$276 \mathrm{mmHg}$ auf $284-366 \mathrm{mmHg}$.

- Die Viruslast nahm ab und war innerhalb von 12 Tagen nach Transfusion nicht mehr nachweisbar (s.u.),

- die SARS-CoV-2-spezifischen ELISA-bestimmten Titer sowie die Neutralisationsantikörpertiter nahmen nach Transfusion zu (40-60 vor und 80-320 an Tag 7 nach Transfusion).

- Das ARDS hatte sich bei 4 Patienten nach 12 Tagen zurückgebildet, $3 \mathrm{~Pa}$ tienten konnten innerhalb von 2 Wochen nach Transfusion von der mechanischen Beatmung genommen werden.

- Patient 2 war zum Zeitpunkt der Transfusion an der ECMO (extrakorporale Membranoxygenierung), diese konnte an Tag 5 nach der Transfusion beendet werden.

- 37 Tage nach Transfusion waren 3 Patienten entlassen worden, 2 Patienten befanden sich noch stationär, aber in stabilem Zustand.

Der Ct-Wert (Threshold Cycle, Schwellenwertzyklus in der Real-Time-quantitativen PCR) bei Krankenhausaufnahme variierte von 18,9-38,0 und am Tag der Plasmatransfusion von 22,0-35,9. Innerhalb eines Tages nach der Transfusion wurde der Wert bei Patient 5 negativ, bei Patient 3 und 4 an Tag 3 und Patient 1 und 2 wurden an Tag 12 nach der Transfusion negativ gemessen. Die antivirale Therapie war kontinuierlich beibehalten worden, bis die Viruslast unter die Nachweisgrenze fiel.

Im Verlauf nach Transfusion nahmen die Entzündungszeichen CRP, Procalcitonin und Interleukin 6 ab. In der CT der Lunge hatten sich bei allen Patienten Zeichen einer schweren Pneumonie gezeigt, welche sich 3 Tage nach der Transfusion bereits in Rückbildung befanden. 
FAZIT

Die Ergebnisse weisen darauf hin, dass Plasma von COVID-19-Genesenen als vielversprechende therapeutische Option dienen kann. Die Therapie wurde gut vertragen und kann möglicherweise das klinische Behandlungsergebnis schwer an COVID-19 Erkrankter durch eine Neutralisierung der Virämie verbessern. Weitere größere, kontrollierte und randomisierte Studien sind erforderlich, um Aussagen zu klinischer Wirksamkeit, optimalem Zeitpunkt und Dosierung treffen zu können, so die Autoren.

Dr. Birgit Gappa, Penzberg 